Os fatores que devem ser considerados para uma efetiva implantação do controle estatístico de processo (CEP): uma revisão de literatura

\title{
The factors that should be considered for an effective implementation of statistical process control (SPC): a literature review
}

Fabiano Rodrigues Soriano ${ }^{1}$, Universidade Estadual de Santa Cruz, Departamento de Ciências Exatas e Tecnológicas

Pedro Carlos Oprime², Universidade Federal de São Carlos, Departamento de Engenharia de Produção

Fabiane Letícia Lizarelli ${ }^{3}$ Universidade Federal de São Carlos, Departamento de Engenharia de Produção

\section{RESUMO}

O Controle Estatístico de Processo (CEP) corresponde a um conjunto de ferramentas estatísticas que visam à melhoria e estabilidade dos padrões da qualidade e é amplamente utilizado pelas como suporte aos programas de qualidade. Ao longo de mais de 50 anos, diversos autores exploraram o assunto apresentando Fatores Críticos para o Sucesso (FCS) para a sua implantação nas indústrias. Contudo, não se encontra na literatura trabalhos que os reúnam e os classifiquem de acordo com dimensões (estatísticas, metodológicas e gerenciais) e níveis de decisão (Estratégico, Tático e Operacional). Esta revisão de literatura é resultado de uma busca aprofundada a respeito dos FCS em trabalhos encontrados nas principais bases de dados disponíveis. Foram identificados e estão listados neste trabalho 44 FCS que devem ser observados para a efetiva implantação do CEP e que possibilitam aos gestores direcionarem seus esforços e recursos. Verifica-se a importância e a carência de estudos que mostrem, efetivamente, como os níveis de decisão e as dimensões interferem no resultado do programa e de que forma os fatores podem corroborar para o sucesso do CEP.

Palavras-chave: Controle Estatístico de Processo. Fatores de Sucesso. Revisão de literatura.

\section{ABSTRACT}

The Statistical Process Control (SPC) corresponds to a set of statistical tools that aim to improve and stabilize quality standards and is widely used as support for quality programs. For more than 50 years, several authors have explored the subject presenting Critical Success Factors (CSF) for its implementation in the industries. However, there are no papers in the literature that gather and classify these factors according to dimensions (statistical, methodological and managerial) and decision levels (Strategic, Tactical and Operational). This literature review is the result of an in-depth search of the CSF in studies found on the main databases available. We identified and listed in this study 44 CSF that must be observed for the effective implementation of the SPC and that enable managers to direct their efforts and resources. The importance and the lack of studies that effectively show how decision levels and dimensions interfere with the program's results and how these factors can corroborate with the success of the SPC, can be verified.

Keywords: Statistical Process Control. Critical Success Factors. Literature review

1. Universidade Estadual de Santa Cruz - Campus Soane Nazaré de Andrade, Rodovia Jorge Amado, Km 16, Bairro Salobrinho, CEP 45662-900. Ilhéus-Bahia, BA, frsoriano@uesc.br; 2. pedro@dep.ufscar.br; 3. fabiane@ dep.ufscar.br

SORIANO, F.R.; OPRIME, P.C.; LIZARELLI, F. L. Os fatores que devem ser considerados para uma efetiva implantação do controle estatístico de processo (CEP): uma revisão de literatura. GEPROS. Gestão da Produção, Operações e Sistemas, v. 15, n. 1, p. $71-91,2020$.

DOI: $10.15675 /$ gepros.v15i1.2259
Editor Responsável: Prof. Dr. Hermes Moretti Ribeiro da Silva 
Os fatores que devem ser considerados para uma efetiva implantação do controle estatístico de processo (CEP): uma revisão de literatura

\section{INTRODUÇÃO}

O Controle Estatístico de Processo (CEP) é descrito, na literatura, como um conjunto de ferramentas bem estabelecidas que visam compreender, monitorar, controlar e melhorar o desempenho dos processos produtivos ao longo do tempo. Sua utilização é fundamental para a estabilização do processo, por meio da identificação das causas de variação. Permite, também, avaliar a estabilidade do processo, uma vez que fornece por meio de gráficos a informação do momento que o processo está fora de controle e a capacidade do processo, pois permite análise em relação aos limites de especificação (ANGHEL, 2001; BHOTE, 1987; LEVINSON, 2011; MONTGOMERY, 2010; OAKLAND, 2008; SORIANO; OPRIME; LIZARELLI, 2017; LIM et. al. 2017).

O CEP tem sido utilizado em processos industriais desde a década de 1920, havendo um aumento do interesse e uso durante a segunda guerra mundial (MONTGOMERY, 2014). O CEP é associado ao uso de gráficos de controle, que permitem o acompanhamento da estabilidade do processo, os primeiros gráficos foram desenvolvidos por Shewhart que englobam o gráfico de média e amplitude e média e desvio padrão (MONTGOMERY, 2014). Lizarelli et al. (2016) realizou uma análise sobre 50 anos (1956 - 2013) de publicação em CEP e a busca na base Web of Science identificou 3,683 artigos no tema, sendo que 62\% foram publicadas na última década de análise (2004-2013), mostrando o interesse e a importância crescente do tema.

O estudo também mostra os principais termos das palavras-chave, permitindo a identificação de tendências de estudo na temática do CEP, como gráficos de controle cumulativos (Cumulative Sum Control Charts - CUSUM), controle estatístico multivariado, gráficos de média móvel ponderada exponencialmente (Exponentially Weighted Moving Average - EWMA) e gráficos não-paramétricos, mostrando o interesse em outros tipos de gráficos que não os tradicionais (LIZARELLI et al., 2016). Outras áreas de interesse que também estão evoluindo englobam gráficos de controle de baixo custo, aplicação do CEP em metrologia, análise da capabilidade do processo e o CEP associado ao controle da qualidade (LIZARELLI et al., 2016).

Apesar do crescente interesse no tema CEP tanto na literatura quanto na prática, e do conceito do CEP ser de simples compreensão, a implantação é complexa (ANTONY; 
Os fatores que devem ser considerados para uma efetiva implantação do controle estatístico de processo (CEP): uma revisão de literatura

BALBONTIN; TANER, 2000), demanda mudanças organizacionais e comportamentais que implicam na necessidade de considerar fatores que transcendem os aspectos técnicos da implantação do CEP (GRIGG; WALLS, 2007). Os fatores que afetam a implantação do CEP, que podem ser chamados de Fatores Críticos de Sucesso (FCS) são complexos e numerosos (ROHANI; YUSOF; MOHAMAD, 2010), a atenção a esses fatores pode aumentar a chance de sucesso de implantação do CEP (ELG; OLSSON; DAHLGAARD, 2008; ROHANI; YUSOF; MOHAMAD, 2010). Por essa razão o tema FCS para implantação e sustentação do CEP tem demandado atenção e interesse.

Diferentes perspectivas de análise dos FCS têm sido utilizadas em estudos sobre CEP, por exemplo, a análise sob as perspectivas de fatores técnicos e organizacionais (DOES; TRIP; SCHIPPERS, 1997; ELG; OLSSON; DAHLGAARD, 2008), ou a separação em gestão do CEP, fator humano e a implantação propriamente dita, envolvendo o uso de ferramentas apropriadas para o monitoramento do processo (XIE; GOH, 1999).

Há diversos exemplos de autores que tentam classificar os FCS em perspectivas, a exemplo de: Soriano, Oprime e Lizarelli (2017); Putri e Yusof, (2009) e Rohani, Yusof e Mohamad, (2010). Contudo, Firka (2011) é pioneiro em apresentar uma forma lógica de agrupar os FCS em três dimensões básicas: Estatística; Metodológica e Gerencial. Apesar do histórico, a literatura estudada sobre o assunto não orienta de forma objetiva o papel de cada nível de decisão na condução das suas atividades do programa e nem como os FCS devem ser trabalhados do ponto de vista estatístico, dos métodos de trabalho ou dos mecanismos gerenciais de apoio.

A dimensão estatística refere-se aos pressupostos estatísticos assumidos, quando da utilização de determinada técnica. Na metodológica, agrupam-se os fatores representados por variáveis relacionadas à condução dos trabalhos e ao aproveitamento das informações geradas pelo sistema de monitoramento, e deve apoiar uma correta intervenção no processo. Por outro lado, a gerencial corresponde aos fatores diretamente influenciados pelos comportamentos e necessidades humanas e precisam ser considerados pelos gestores (MONTGOMERY, 2010; FIRKA, 2011; SORIANO; OPRIME; LIZARELLI, 2017).

De maneira complementar, Soriano (2015) divide estes FCS para implantação do CEP, de acordo com os níveis hierárquicos decisórios da organização, o que possibilita uma efetiva distribuição de responsabilidade. Assim, tais dimensões e responsabilidades devem de 
Os fatores que devem ser considerados para uma efetiva implantação do controle estatístico de processo (CEP): uma revisão de literatura

ser observadas e consideradas de igual forma no processo de implantação do CEP (SORIANO; OPRIME; LIZARELLI, 2017).

O objetivo deste trabalho é apresentar os FCS, a partir de uma análise da literatura dos principais trabalhos que abordam este assunto, agrupá-los de acordo com as dimensões propostas por Firka (2011) e consoante os níveis de responsabilidade, apresentados por Soriano (2015), possibilitando de uma maneira bem prática aos gestores a observação de possíveis deficiências em seus programas CEP.

Dessa forma, compreender como os FCS para a implantação do CEP estão relacionados aos níveis de decisão da empresa, pode auxiliar os gestores a desdobrarem corretamente as metas de implantação e de sustentação do programa CEP, bem como acompanhar o processo de implantação de uma maneira mais eficiente (AHMAD, 2012; AHMED; KHAN; GHOSH, 2012; ANTONY; BALBONTIN; TANER, 2000; ANTONY; TANER, 2003; BEVILACQUA, 2011; GORDON, 1994, LIM; ANTONY, 2013; PUTRI; YUSOF, 2009; RANTAMÄKI; TIAINEN; KÄSSI, 2013; SANTANA Jr, 2014).

Os principais FCS para a implantação do CEP são bem conhecidos na literatura e mesmo de forma empírica, no entanto, algumas organizações ainda não conseguem direcionar esforços para que os FCS sejam utilizados de forma correta, seja por desconhecerem o nível hierárquico que deve se responsabilizar pelo FCS, por atribuírem responsabilidade indevida, ou ainda por desconsiderarem alguns dos fatores, relacionado às especificidades de seu processo produtivo e importantes (AHMAD, 2012; ANGHEL, 2001; ASQ/AIAG, 2002, 2005; BEVILACQUA, 2011; BHOTE, 1987; CASTAGLIOLA, 2009, 2013; HRADESKY, 1989; LIM; ANTONY; ALBLIWI, 2014; NOSKIEVICOVA; PAUCHOVA; KELBLEROVA，2011; PUTRI; YUSOF，2009; RUPA; EVANS，2012; SORIANO; OPRIME; LIZARELLI, 2017; LIM et. al. 2017).

\section{MÉTODO DE PESQUISA}

O método utilizado para o trabalho foi uma revisão bibliográfica sistematizada com a adição de outras fontes via referência cruzada. A revisão bibliográfica sistemática (RBS) envolve, primeiramente, um planejamento da pesquisa, uma das principais etapas da pesquisa, pois, é no planejamento que se definem a questão a ser respondida com a revisão e os objetivos da RBS. Também é nessa etapa que são definidas as palavras-chaves (strings) pelas 
Os fatores que devem ser considerados para uma efetiva implantação do controle estatístico de processo (CEP): uma revisão de literatura

quais os trabalhos acadêmicos serão localizados; as fontes primárias (bases de dados acadêmicos), nas quais se farão as buscas pelos trabalhos acadêmicos; os critérios de inclusão e/ou exclusão dos trabalhos acadêmicos encontrados, pelos quais se fará a avaliação crítica, determinando-se quais trabalhos devem ser incluídos ou excluídos da amostra obtida; e os critérios de qualificação, pelos quais estudos localizados serão analisados (CONFORTO; AMARAL; SILVA, 2011; BRERETON et al., 2007).

Após o planejamento, o processamento do que foi planejado leva à localização de trabalhos acadêmicos no tema proposto, que, em seguida, passam por filtros para refinamento da pesquisa, por meio da aplicação de critérios de inclusão, exclusão e qualificação, visando identificar trabalhos relevantes para o objetivo proposto e eliminar os que não aderem ao escopo da questão de pesquisa. A última etapa é a análise da saída, cujo objetivo é realizar a síntese dos resultados e propor, caso pertinente, modelos teóricos (CONFORTO; AMARAL; SILVA, 2011). A Figura 1 apresenta as etapas propostas.

Figura 1 - Modelo para condução de revisão bibliográfica sistemática

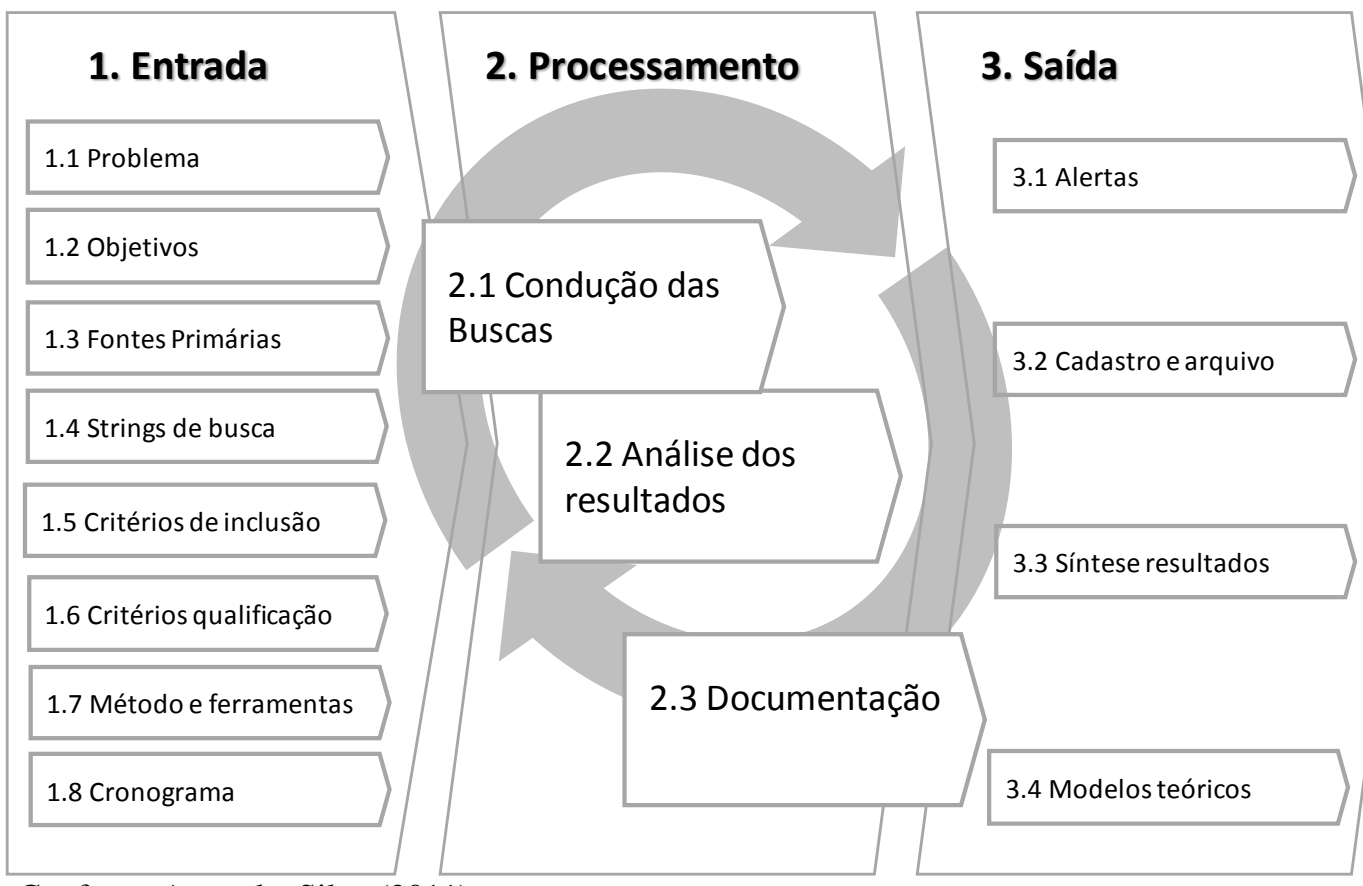

Fonte: Conforto, Amaral e Silva (2011).

Os resultados da busca são apresentados no Quadro 1. 
Os fatores que devem ser considerados para uma efetiva implantação do controle estatístico de processo (CEP): uma revisão de literatura

Quadro 1 - Etapas da Revisão Bibliográfica Sistemática

\begin{tabular}{|c|c|}
\hline \multicolumn{2}{|c|}{ Planejamento } \\
\hline Questão de Pesquisa & $\begin{array}{l}\text { Quais são os Fatores Críticos de Sucesso para a } \\
\text { implantação e sustentação do Controle Estatístico do } \\
\text { Processo? }\end{array}$ \\
\hline String de busca & $\begin{array}{l}\text { (("statistical process control"or"SPC"or"control } \\
\text { chart") and ("critical success factors"or"success } \\
\text { factors")) em inglês; ou (("controle estatístico do } \\
\text { processo"or"CEP"or"carta de controle") and ("fatores } \\
\text { críticos de sucesso"or"fatores de sucesso")) em } \\
\text { português. }\end{array}$ \\
\hline Bases de dados & Web of Science, Scopus, Engineering Village e Scielo \\
\hline Busca & Título, resumo e palavras-chave \\
\hline Período da busca & $1960-2017$ \\
\hline Critérios de inclusão & $\begin{array}{l}\text { Artigos de congresso e revistas que apresentem FCS } \\
\text { para a implantação ou sustentação do CEP. }\end{array}$ \\
\hline Critérios de exclusão & $\begin{array}{l}\text { Trabalhos que tratem de FCS para outros programas } \\
\text { que não o CEP; trabalhos que não tratam diretamente } \\
\text { de FCS. }\end{array}$ \\
\hline Critérios de qualificação & $\begin{array}{l}\text { A busca procura pelos próprios FCS e esses é que são } \\
\text { alvos de análise e qualificação. }\end{array}$ \\
\hline \multicolumn{2}{|l|}{ Execução } \\
\hline Número de trabalhos retornados & $\begin{array}{l}\text { Web of Science (9), Scopus (25) e Engineering Village } \\
(11), \text { Scielo (0) }\end{array}$ \\
\hline $\begin{array}{l}\text { Número de artigos selecionados após os critérios de } \\
\text { inclusão e exclusão }\end{array}$ & $\begin{array}{l}\text { Web of Science (3), Scopus (8), Engineering Village } \\
\text { (4) }\end{array}$ \\
\hline $\begin{array}{l}\text { Número de artigos selecionados excluindo-se os } \\
\text { repetidos }\end{array}$ & 8 \\
\hline
\end{tabular}

Fonte: Próprios autores (2018).

Os artigos selecionados pelas buscas englobam: Lim et al., 2017; Soriano, Oprime e Lizarelli (2017); Antony, Lim e Arshed (2016); Lim e Antony (2013); Mahanti e Evans (2012); Rohani, Yusof e Mohamad (2009); Rungasamy, Antony e Ghosh (2002); Antony e Taner (2003). Como as buscas retornaram um baixo número de artigos nas bases analisadas, o que indica necessidades de trabalhos acadêmicos neste tema, outros trabalhos foram encontrados por meio de referência cruzada, ou seja, são artigos que foram citados por um dos oito artigos acima apresentados.

\section{FATORES CRÍTICOS DE SUCESSO PARA IMPLANTAÇÃo E MANUTENÇÃo DO CEP}

Neste tópico é realizada a compilação dos FCS identificados na literatura considerando três níveis de decisão e responsabilidade industrial (estratégico, tático e GEPROS. Gestão da Produção, Operações e Sistemas, v. 15, nº 1, p. 71- 91, 2020. 
Os fatores que devem ser considerados para uma efetiva implantação do controle estatístico de processo (CEP): uma revisão de literatura

operacional. Os FCS também são subdivididos de acordo com as dimensões propostas por Firka (2011), gerencial, metodológica e estatística.

O Quadro2 apresenta os FCS relacionados ao nível estratégico. Os FCS aqui descritos estão diretamente ao apoio adequado da alta administração à implantação do CEP. Os Fatores da dimensão gerencial estão relacionados ao auxílio que a alta administração pode prover e as ações gerenciais para executá-lo, enquanto a dimensão metodológica está direcionada à ações estratégicas para aplicação do CEP. Percebe-se que não há fatores da dimensão operacional, já que o nível estratégico estaria mais direcionado para ações globais da organização.

Quadro 2 - Fatores críticos de sucesso relacionados ao nível estratégico

\begin{tabular}{|c|c|c|}
\hline Dimensão & FCS & Fontes \\
\hline \multirow{10}{*}{$\begin{array}{l}\frac{\pi}{0} \\
\frac{\pi}{0} \\
\overline{0} \\
0 \\
0\end{array}$} & $\begin{array}{l}\text { 1.1 Comprometimento da alta } \\
\text { administração }\end{array}$ & $\begin{array}{l}\text { ANTONY; BALBONTIN; TANER, 2000; MONTGOMERY, } \\
\text { 2004; GRIGG e WALLS, 2007; OAKLAND, 2008; HOERL; } \\
\text { SNEE, 2010; RUPA; EVANS, 2012; SHARMA; KHARUB, } \\
\text { 2014; TOLEDO; et.al., 2017 }\end{array}$ \\
\hline & $\begin{array}{l}\text { 1.2 Integração dos setores da } \\
\text { empresa }\end{array}$ & $\begin{array}{l}\text { HAYES e ROMIG, 1977; ANTONY; BALBONTIN; TANER, } \\
\text { 2000; MONTGOMERY, 2004; GRIGG E WALLS, 2007; } \\
\text { OAKLAND, 2008; HOERL, SNEE, 2010. SHARMA; } \\
\text { KHARUB, 2014. }\end{array}$ \\
\hline & 1.3 Treinamento Gerencial & $\begin{array}{l}\text { HAYES e ROMIG, 1977; ANTONY; BALBONTIN; TANER, } \\
\text { 2000; MONTGOMERY, 2004; GRIGG e WALLS, 2007; } \\
\text { OAKLAND, 2008; HOERL, SNEE, 2010; NOSKIEVICOVA; } \\
\text { PAUCHOVA; KELBLEROVA, 2011; SHARMA; KHARUB, } \\
\text { 2014. TOLEDO; et.al., 2017. }\end{array}$ \\
\hline & $\begin{array}{l}\text { 1.4 Desenvolvimento da } \\
\text { melhoria contínua }\end{array}$ & $\begin{array}{l}\text { AHMAD et. al., 2012; RUPA; EVANS, 2012; GRAÇA; et. al., } \\
\text { 2014; SHARMA; KHARUB, 2014; TOLEDO; et.al., } 2017 .\end{array}$ \\
\hline & $\begin{array}{l}\text { 1.5 Alinhamento aos } \\
\text { objetivos estratégicos }\end{array}$ & $\begin{array}{l}\text { ANTONY; BALBONTIN; TANER, 2000; MONTGOMERY, } \\
\text { 2004; GRIGG e WALLS, 2007; OAKLAND, 2008; HOERL, } \\
\text { SNEE, 2010; GRAÇA et. al., 2014; TOLEDO; et.al., } 2017 .\end{array}$ \\
\hline & 1.6 Infraestrutura & $\begin{array}{l}\text { BHOTE, 1987; PETERSEN, 2011; AHMED; KHAN; GHOSH, } \\
\text { 2012; MUJTABA; FELDT; MAHANTI; EVANS, 2012; } \\
\text { SHARMA; KHARUB; 2014; LIM et. al. 2017; TOLEDO; et.al., } \\
2017 .\end{array}$ \\
\hline & 1.7 Mudança cultural & $\begin{array}{l}\text { HAYES e ROMIG, 1977; ANTONY; BALBONTIN; TANER, } \\
\text { 2000; MONTGOMERY, 2004; GRIGG e WALLS, 2007; } \\
\text { PHYANTHAMILKUMARAN; } \quad \text { FERNANDO, } \\
\text { OAKLAND, 2008; HOERL, SNEE, 2010; MAHANTI e } \\
\text { EVANS; 2012; LIM e ANTONY, 2013; GRAÇA et. al. 2014; } \\
\text { TOLEDO; et.al., 2017. }\end{array}$ \\
\hline & 1.8 Envolvimento de todos & $\begin{array}{l}\text { HAYES e ROMIG, 1977,ANTONY; BALBONTIN; TANER, } \\
\text { 2000; SHARMA; KHARUB, 2014; OAKLAND, 2008; } \\
\text { MONTGOMERY, 2004; GRIGG e WALLS, 2007; HOERL; } \\
\text { SNEE, 2010; LIM et. al. 2017; TOLEDO; et.al., 2017. }\end{array}$ \\
\hline & $\begin{array}{l}\text { 1.9 Utilização do suporte } \\
\text { externo }\end{array}$ & $\begin{array}{l}\text { GRIGG e WALLS, 2007; MONTGOMERY, 2004; OAKLAND, } \\
\text { 2008; HOERL, SNEE, 2010; RANTAMÄKI; TIAINEN; KÄSSI; } \\
\text { 2013. }\end{array}$ \\
\hline & $\begin{array}{l}1.10 \text { Ênfase do controle em } \\
\text { substituição às inspeções }\end{array}$ & $\begin{array}{l}\text { MONTGOMERY, 2004; GRIGG e WALLS, 2007; OAKLAND, } \\
\text { 2008; HOERL, SNEE, 2010, LIM; ANTONY; ALBLIWI, 2014; }\end{array}$ \\
\hline
\end{tabular}


Os fatores que devem ser considerados para uma efetiva implantação do controle estatístico de processo (CEP): uma revisão de literatura

\begin{tabular}{|c|c|c|}
\hline & & SHARMA; KHARUB, 2014. \\
\hline \multirow{5}{*}{$\begin{array}{l}\tilde{0} \\
.00 \\
0 \\
0 \\
0 \\
0 \\
0 \\
\sum \\
\Sigma\end{array}$} & $\begin{array}{l}\text { 1.11 Aplicação dos conceitos } \\
\text { do Pensamento Estatístico }\end{array}$ & $\begin{array}{l}\text { GRIGG E WALLS, 2007; AHMAD et. al., 2012; RANTAMÄKI; } \\
\text { TIAINEN; KÄSSI; 2013; GRAÇA; et. al., 2014; SORIANO e } \\
\text { OPRIME, 2014; SHARMA; KHARUB, 2014; TOLEDO; et.al., } \\
2017 .\end{array}$ \\
\hline & $\begin{array}{l}\text { 1.12 Estipulação de metas de } \\
\text { desempenho do processo }\end{array}$ & $\begin{array}{l}\text { SNEE, 1990; GRIGG; WALLS, 2007; HOERL, SNEE, 2010; } \\
\text { TOLEDO; et.al, } 2017 .\end{array}$ \\
\hline & $\begin{array}{l}1.13 \text { Desenvolvimento de um } \\
\text { plano adequado de } \\
\text { implantação }\end{array}$ & $\begin{array}{l}\text { MONTGOMERY, 2004; GRIGG E WALLS, 2007; OAKLAND, } \\
\text { 2008; HOERL, SNEE, 2010 RUPA; EVANS, 2012; LIM; } \\
\text { ANTONY, 2014; SHARMA; KHARUB, 2014. }\end{array}$ \\
\hline & $\begin{array}{l}\text { 1.14 Avaliação dos custos de } \\
\text { implantação }\end{array}$ & $\begin{array}{l}\text { MUJTABA; FELDT; PETERSEN, 2011; AHMED; KHAN; } \\
\text { MAHANTI; EVANS, 2012; GHOSH, 2012; LIM e ANTONY, } \\
\text { 2013 LIM et. al. 2017. }\end{array}$ \\
\hline & $\begin{array}{l}\text { 1.15 Utilizar o CEP como } \\
\text { ferramenta para a melhoria da } \\
\text { Qualidade }\end{array}$ & $\begin{array}{l}\text { MONTGOMERY, 2004; GRIGG e WALLS, 2007; OAKLAND, } \\
\text { 2008; HOERL, SNEE, 2010; RUPA; EVANS, 2012; LIM; } \\
\text { ANTONY, 2014; SHARMA; KHARUB, 2014. }\end{array}$ \\
\hline
\end{tabular}

Fonte: Próprios autores (2018).

Do ponto de vista das competências do nível estratégico para a implantação do CEP, faz-se necessário haver o comprometimento da alta administração por meio de ações que fomentem e motivem os demais níveis da organização em busca da implantação eficiente, vislumbrando a integração entre os setores e o estabelecimento da infraestrutura necessária para a operacionalização das atividades. Tal fato inclui a necessidade de treinamento gerencial, bem como o alinhamento aos objetivos estratégicos do CEP por meio de iniciativas de melhoria da qualidade. Uma boa prática apresentada pela literatura diz respeito à utilização de empresas de consultoria para viabilizar o desenvolvimento de um plano adequado de implantação. Neste contexto, os custos do serviço e implantação devem ser observados. (AHMED; KHAN; GHOSH, 2012; MUJTABA; FELDT; MAHANTI; EVANS, 2012; LIM et. al. 2017).

Os FCS pertencentes ao nível estratégico e classificados dentro da dimensão gerencial são variáveis relacionadas à motivação para a mudança dentro da organização, sendo fundamental que o processo de implantação das cartas de controle seja decorrente de necessidades e com o envolvimento de todos, e não apenas de um modismo de gestão (CASTAGLIOLA, 2009; 2013; CASTAGLIOLA; MARAVELAKIS, 2009; WOODALL, 2000; MONTGOMERY; WOODALL, 1999).

O nível estratégico deve reconhecer a existência da variabilidade no sistema produtivo e buscar sua redução de forma constante e sistemática. Assim, é imprescindível enfatizar a necessidade do estabelecimento do controle sobre o processo, reduzindo-se sistematicamente a importância das realizações de inspeções como mecanismo de reconhecimento de garantia GEPROS. Gestão da Produção, Operações e Sistemas, v. 15, nº 1, p. 71- 91, 2020 
Os fatores que devem ser considerados para uma efetiva implantação do controle estatístico de processo (CEP): uma revisão de literatura

da qualidade e, de fato, acreditar no potencial do CEP como ferramenta para monitorar o processo e de garantia da qualidade, enfatizados pelos Fatores da dimensão metodológica, que saliente também um plano adequado para a implantação (CASTAGLIOLA, 2009; 2013; CASTAGLIOLA; MARAVELAKIS, 2009; PHYANTHAMILKUMARAN; FERNANDO, 2008; MAHANTI; EVANS, 2012; LIM; ANTONY, 2013; RANTAMÄKI; TIAINEN; KÄSSI, 2013; SORIANO; OPRIME, 2014; MUJTABA; FELDT; PETERSEN, 2011; LIM; ANTONY, 2013; LIM et. al. 2017).

No Quadro 3 estão alocados os fatores críticos, cuja responsabilidade pela implantação pode ser atribuída ao nível tático, que deve conhecer profundamente os conceitos do CEP, bem como as características do produto e do processo. Percebe-se que estes fatores críticos estão distribuídos nas três dimensões propostas por Firka (2011). Tal fato corrobora que o nível tático tem atribuições relevantes em relação ao CEP, ao envolver decisões que estão relacionados ao auxílio no suporte às atividades no nível operacional, sendo responsável pelo desdobramento e alinhamento dessas metas com os objetivos estratégicos das organizações, ou seja, é responsável por conectar o nível estratégico ao operacional e comunicar e desdobrar as ações do nível estratégico ao nível operacional (GRIGG; WALLS, 2007; HOERL, SNEE, 2010; LIM et. al. 2017).

Quadro 3 - Fatores críticos de sucesso relacionados ao nível tático

\begin{tabular}{|c|c|c|}
\hline Dimensão & FCS & Fontes \\
\hline & $\begin{array}{l}2.1 \text { Aplicação dos } \\
\text { conceitos da Gestão } \\
\text { da Qualidade Total } \\
\text { (TQM) }\end{array}$ & $\begin{array}{l}\text { MONTGOMERY, 2004; GRIGG E WALLS, 2007; OAKLAND, 2008; } \\
\text { HOERL, SNEE, 2010; AHMAD et. al., 2012; GRAÇA; et. al., 2014; } \\
\text { SHARMA; KHARUB; 2014; LIM et. al. 2017; TOLEDO; et.al., 2017. }\end{array}$ \\
\hline & $\begin{array}{lr}2.2 & \text { Governança } \\
\text { para } & \text { armazenar } \\
\text { dados } & \end{array}$ & $\begin{array}{l}\text { GROOVER, 2007; RUPA; EVANS, 2012; SORIANO e OPRIME, 2014; } \\
\text { TOLEDO; et.al., 2017. }\end{array}$ \\
\hline & $\begin{array}{l}2.3 \text { Resistência à } \\
\text { mudança }\end{array}$ & $\begin{array}{llccc}\text { BHOTE; 1987; } & \text { ANTONY; } & \text { BALBONTIN; } & \text { TANER, } & \text { 2000; } \\
\text { MONTGOMERY, 2014; GRIGG e } & \text { WALLS, 2007; OAKLAND, 2008; } \\
\text { HOERL, SNEE, 2010; SHARMA; KHARUB, 2014. }\end{array}$ \\
\hline & $\begin{array}{l}2.4 \text { Simplificação } \\
\text { da abordagem }\end{array}$ & $\begin{array}{l}\text { MUJTABA; FELDT; PETERSEN, 2011; AHMED; KHAN; GHOSH, 2012; } \\
\text { MAHANTI; EVANS, 2012; LIM e ANTONY, 2013; SHARMA; KHARUB; } \\
\text { 2014. }\end{array}$ \\
\hline & $\begin{array}{l}2.5 \text { Treinamento de } \\
\text { reciclagem }\end{array}$ & $\begin{array}{l}\text { HAYES e ROMIG, 1977, ANTONY; BALBONTIN; TANER, 2000; } \\
\text { NOSKIEVICOVA; PAUCHOVA; KELBLEROVA, 2011; RUPA; EVANS, } \\
\text { 2012; LIM et. al. 2017; TOLEDO; et.al., 2017. }\end{array}$ \\
\hline $\begin{array}{l}\frac{\pi}{0} \\
\frac{0}{0} \\
\frac{0}{0} \\
0\end{array}$ & $\begin{array}{ll}2.6 \text { Utilização } & \text { de } \\
\text { mecanismo } & \text { de } \\
\text { reconhecimento } & \text { do } \\
\text { sucesso } & \\
\end{array}$ & $\begin{array}{l}\text { MONTGOMERY, 2014; ANTONY; BALBONTIN; TANER, 2000; GRIGG } \\
\text { e WALLS, 2007; OAKLAND, 2008; HOERL, SNEE, } 2010 \text { RUPA; EVANS, } \\
\text { 2012; SORIANO e OPRIME, 2014; LIM et. al. 2017. }\end{array}$ \\
\hline 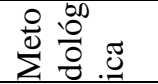 & $\begin{array}{lr}2.7 & \text { Não } \\
\text { consideração } & \text { do }\end{array}$ & $\begin{array}{l}\text { BHOTE, 1987; MUJTABA; FELDT; PETERSEN, 2011; AHMED; KHAN; } \\
\text { GHOSH, 2012; MAHANTI; EVANS, 2012; RUPA; EVANS, 2012; LIM e }\end{array}$ \\
\hline
\end{tabular}


Os fatores que devem ser considerados para uma efetiva implantação do controle estatístico de processo (CEP): uma revisão de literatura

\begin{tabular}{|c|c|c|}
\hline & \begin{tabular}{|lr} 
operador & como \\
controlador & do \\
processo & \\
\end{tabular} & $\begin{array}{l}\text { ANTONY, 2013; SHARMA; KHARUB, 2014; LIM et. al. 2017; TOLEDO; } \\
\text { et.al., 2017. }\end{array}$ \\
\hline & $\begin{array}{l}2.8 \text { Controle de } \\
\text { características } \\
\text { necessárias do } \\
\text { processo/produto }\end{array}$ & $\begin{array}{l}\text { ANTONY; BALBONTIN; TANER, 2000; MONTGOMERY, 2004; GRIGG } \\
\text { e WALLS, 2007; OAKLAND, 2008; HOERL, SNEE, 2010; RUPA; } \\
\text { EVANS, 2012; SHARMA; KHARUB, 2014; LIM et. al. 2017; TOLEDO; } \\
\text { et.al., 2017. }\end{array}$ \\
\hline & $\begin{array}{lll}2.9 & \text { Uso } & \mathrm{de} \\
\text { Facilitadores } & \end{array}$ & $\begin{array}{l}\text { HAYES e ROMIG, 1977, ANTONY; BALBONTIN; TANER, 2000; } \\
\text { MONTGOMERY, 2004; GRIGG e WALLS, 2007; OAKLAND, 2008; } \\
\text { HOERL; SNEE, 2010; RUPA; EVANS, 2012, SHARMA; KHARUB, 2014; } \\
\text { TOLEDO; et.al., 2017. }\end{array}$ \\
\hline & \begin{tabular}{|l|}
2.10 Evitar excesso \\
de cartas de \\
controle
\end{tabular} & $\begin{array}{l}\text { MONTGOMERY, 2004; GRIGG e WALLS, 2007; OAKLAND, 2008; } \\
\text { HOERL; SNEE, 2010; AHMAD et. al., 2012; GRAÇA; et. al., 2014; } \\
\text { SHARMA; KHARUB; 2014. }\end{array}$ \\
\hline & \begin{tabular}{|lr|}
\multicolumn{2}{|l|}{.11 Documentação } \\
atualizada & dos \\
parâmetros & de \\
processo & \\
\end{tabular} & $\begin{array}{l}\text { ANTONY; BALBONTIN; TANER, 2000; MONTGOMERY, 2004; GRIGG } \\
\text { e WALLS, 2007; OAKLAND, 2008; HOERL, SNEE, 2010; RUPA; } \\
\text { EVANS, 2012; SORIANO e OPRIME, 2014; TOLEDO; et.al., 2017. }\end{array}$ \\
\hline & $\begin{array}{l}2.12 \text { Uso de piloto } \\
\text { na implantação }\end{array}$ & $\begin{array}{l}\text { ANTONY; BALBONTIN; TANER, 2000; MONTGOMERY, 2004; GRIGG } \\
\text { e WALLS, 2007; OAKLAND, 2008; HOERL, SNEE, 2010; SHARMA; } \\
\text { KHARUB, 2014; TOLEDO; et.al., 2017. }\end{array}$ \\
\hline & $\begin{array}{l}2.13 \text { Planejamento } \\
\text { da implantação do } \\
\text { CEP }\end{array}$ & $\begin{array}{l}\text { MONTGOMERY, 2004; GRIGG e WALLS, 2007; OAKLAND, 2008; } \\
\text { HOERL; SNEE, 2010; AHMAD et. al., 2012; GRAÇA; et. al., 2014; } \\
\text { SHARMA; KHARUB; 2014. }\end{array}$ \\
\hline & $\begin{array}{l}2.14 \text { Verificação da } \\
\text { eficiência do } \\
\text { sistema de medição }\end{array}$ & $\begin{array}{l}\text { ASQ/AIAG, 2002; MONTGOMERY, 2004; GRIGG e WALLS, 2007; } \\
\text { OAKLAND, 2008; HOERL, SNEE, 2010; SHARMA; KHARUB, } 2014 \text { LIM } \\
\text { et. al. 2017. }\end{array}$ \\
\hline & $\begin{array}{l}2.15 \text { Seleção correta } \\
\text { das cartas de } \\
\text { controle }\end{array}$ & $\begin{array}{l}\text { ANTONY; BALBONTIN; TANER, 2000; MONTGOMERY, 2004; GRIGG } \\
\text { e WALLS, 2007; OAKLAND, 2008; HOERL, SNEE, 2010; RUPA; } \\
\text { EVANS, 2012; TOLEDO; et.al., 2017. }\end{array}$ \\
\hline & \begin{tabular}{|l|}
2.16 Busca pelo \\
conhecimento \\
estatístico
\end{tabular} & $\begin{array}{l}\text { ANTONY; BALBONTIN; TANER, 2000; MONTGOMERY, 2004; GRIGG } \\
\text { e WALLS, 2007; OAKLAND, 2008; HOERL, SNEE, 2010; RUPA; } \\
\text { EVANS, 2012 LIM; ANTONY, 2014; LIM et. al. 2017. }\end{array}$ \\
\hline 5 & $\begin{array}{l}2.17 \text { Atuação nas } \\
\text { causas de variações }\end{array}$ & $\begin{array}{l}\text { GRIGG e WALLS, 2007; MONTGOMERY, 2004; OAKLAND, 2008; } \\
\text { HOERL, SNEE, 2010; MONTGOMERY, 2010; AHMAD et. al., 2012; } \\
\text { SHARMA; KHARUB, 2014; TOLEDO; et.al., 2017. }\end{array}$ \\
\hline
\end{tabular}

Fonte: Próprios autores (2018).

O nível tático apresenta uma interface entre os níveis, e é de sua responsabilidade transformar os objetivos estratégicos da organização em ações concretas. A literatura estudada salienta que para uma efetiva implantação do CEP o nível tático em suas ações gerenciais, deve promover os conceitos de qualidade, principalmente os relacionados à melhoria da qualidade. Tal procedimento auxiliará o direcionamento das atividades do CEP, bem como a fixação de conceitos básicos no nível operacional (GRIGG; WALLS, 2007; GROOVER, 2007; HAYES; ROMIG, 1977; HOERL; SNEE, 2010; LIM; ANTONY, 2013; MAHANTI; EVANS, 2012; MONTGOMERY, 2004; MONTGOMERY, 2010; LIM et. al. 2017).

Um problema recorrente em relação ao lançamento do CEP diz respeito à resistência em relação à mudança por parte do nível operacional às novas atividades. Neste sentido, cabe GEPROS. Gestã̃o da Produção, Operações e Sistemas, v. 15, nº 1, p. 71- 91, 2020. 
Os fatores que devem ser considerados para uma efetiva implantação do controle estatístico de processo (CEP): uma revisão de literatura

ao nível tático em suas ações gerenciais, simplificar a implantação para o nível operacional com treinamentos e utilização de mecanismo de reconhecimento do sucesso dos operadores engajados no CEP (MONTGOMERY, 2014; SHARMA; KHARUB, 2014; SORIANO; OPRIME, 2014).

É importante que existam eventos dentro da organização, premiando boas práticas na implantação do programa CEP, além dos resultados obtidos por meio da utilização destes. Os treinamentos de reciclagem ajudam os operadores a manterem a atenção na estabilidade do processo, além de proporcionar o conhecimento estatístico, necessário à condução das atividades (ANTONY; BALBONTIN; TANER, 2000; 2010; LIM; ANTONY, 2013; SHAININ, 1996; WOODALL, 2000; MONTGOMERY; WOODALL, 1999; LIM et. al. 2017).

O nível tático deve considerar o operador como parte fundamental do processo de implantação do CEP, devido a sua responsabilidade em coletar dados e fazer intervenções necessárias para que este retorne a sua estabilidade. Assim, os responsáveis pelo nível tático devem disponibilizar a figura de um facilitador para que este auxilie e cobre a utilização das técnicas (AHMAD et. al., 2012; AHMED; KHAN; GHOSH, 2012; ANTONY; BALBONTIN; TANER, 2000; GHOSH, 2012; HOERL; SNEE, 2010; LIM ; ANTONY, 2013; MAHANTI; EVANS, 2012).

Em relação às ações metodológicas, a implantação deve ser realizada em etapas, com planejamento e realizar um projeto piloto para demonstrar os benefícios alcançados, sempre vislumbrando uma futura aplicação do CEP em outras partes do processo, de forma gradativa (MUJTABA; FELDT; PETERSEN, 2011; NOSKIEVICOVA; PAUCHOVA; KELBLEROVA, 2011; OAKLAND, 2008; RUPA; EVANS, 2012; SHARMA; KHARUB, 2014; SORIANO; OPRIME, 2014.

Cabe ao nível tático, em relação à dimensão metodológica, fornecer e zelar pela documentação atualizada dos parâmetros do produto e processo; isso possibilitará o acompanhamento mais preciso das causas de variação. É importante que os dados sejam armazenados adequadamente, assim quando houver a necessidade de recuperar algum dado histórico, este seja de fácil acesso para que, também, seja possível compreender as circunstâncias de sua ocorrência (SHARMA; KHARUB, 2014).

A literatura a respeito da verificação da eficiência do sistema de medição é recorrente, desta forma, o nível tático deve zelar e buscar garantias que visem minimizar a variabilidade GEPROS. Gestão da Produção, Operações e Sistemas, v. 15, nº 1, p. 71- 91, 2020. 
Os fatores que devem ser considerados para uma efetiva implantação do controle estatístico de processo (CEP): uma revisão de literatura

no sistema de medição. Consequentemente, a maior parte da variação encontrada no processo deve ser oriunda do sistema produtivo (ANTONY; BALBONTIN; TANER, 2000; ASQ/AIAG, 2004; GRIGG; WALLS, 2007; HAYES; ROMIG, 1977; HOERL; SNEE, 2010; LIM; ANTONY, 2013; MAHANTI; EVANS, 2012; MONTGOMERY, 2010; MUJTABA; FELDT; PETERSEN, 2011; NOSKIEVICOVA; PAUCHOVA; KELBLEROVA， 2011; OAKLAND, 2008; RUPA; EVANS, 2012; SHARMA; KHARUB, 2014).

Ainda em relação à dimensão metodológica, alguns autores advertem sobre os problemas de utilização de um excesso de cartas de controle e do controle de características desnecessárias, bem como da seleção correta dos tipos adequados de carta de controle. O nível tático deve, efetivamente, conhecer as diferentes cartas de controle e fazer a seleção apropriada. O excesso de controle de característica pode levar a uma ineficiência na observação das relações entre as causas de variação, além de ser um trabalho desnecessário (SHAININ，1996; WOODALL，2000; MONTGOMERY; WOODALL，1999; SORIANO; OPRIME; LIZARELLI, 2017; LIM et. al. 2017).

Considerando à dimensão estatística, há ações que necessitam do apoio e experiência do nível tático para a implantação, para que, posteriormente se tornem ações operacionais. A primeira delas está relacionada com a seleção correta das cartas de controle. Montgomery (2004) apresenta um guia para selecionar a carta de controle adequada de acordo com as características do produto e processo e da característica da qualidade a ser analisada. As principais cartas de controle para variáveis são as $\bar{X} / \mathrm{R} ; \bar{X} / \mathrm{S}$, de medidas individuais e CUSUM. Já para atributos destaca-se as $p, n p, c, u$. Além disso existem cartas não paramétricas e multivariadas.

A razão principal da utilização das cartas de controle é, justamente, identificar e atuar nas causas de variação do processo. Assim, o nível tático deve reconhecer e aplicar de forma correta as cartas de controle para que se possa, efetivamente, agir na redução das causas de variação, para isso é necessária a busca constante de conhecimento estatístico (CASTAGLIOLA, 2009; 2013; CASTAGLIOLA; MARAVELAKIS, 2009; LIM et. al. 2017).

No Quadro 4 estão elencados os fatores, cuja responsabilidade está mais relacionada ao nível operacional, que envolve a condução das atividades práticas do cotidiano das empresas. De maneira geral, os FCS são predominantemente de dimensão estatística, de 
Os fatores que devem ser considerados para uma efetiva implantação do controle estatístico de processo (CEP): uma revisão de literatura

acordo com a proposta de dimensões de Firka, (2011). Entretanto, como recomenda a literatura, são de responsabilidade do nível operacional, fatores metodológicos tais como: ajustes no processo e tomada de decisão sobre o processo.

Quadro 4 - Fatores críticos de sucesso relacionados ao nível operacional

\begin{tabular}{|c|c|c|}
\hline Dimensão & FCS & Fontes \\
\hline \multirow[b]{4}{*}{$\begin{array}{l}\frac{\pi}{0} \\
.0 \\
0 \\
0 \\
0 \\
0 \\
0 \\
0 \\
\sum^{0}\end{array}$} & $\begin{array}{l}\text { 3.1 Agilidade na } \\
\text { identificação e resolução } \\
\text { de problemas }\end{array}$ & $\begin{array}{l}\text { BHOTE, 1987; MUJTABA; FELDT; PETERSEN, 2011; } \\
\text { MAHANTI; EVANS, 2012; AHMED; KHAN; GHOSH, 2012; } \\
\text { LIM e ANTONY, 2013; SHARMA; KHARUB, } 2014 .\end{array}$ \\
\hline & $\begin{array}{l}3.2 \text { Utilização de } \\
\text { pacotes computacionais }\end{array}$ & $\begin{array}{l}\text { AHMED; KHAN; GHOSH, 2012; MUJTABA; FELDT; } \\
\text { MAHANTI; EVANS, 2012; RUPA; LIM e ANTONY, 2013; } \\
\text { SHARMA; KHARUB; 2014; AB-RAHMAN, et. al., 2015; } \\
\text { TOLEDO; et.al., 2017. }\end{array}$ \\
\hline & $\begin{array}{l}\text { 3.3 Conhecimento dos } \\
\text { conceitos estatísticos, } \\
\text { relacionados ao CEP }\end{array}$ & $\begin{array}{l}\text { BHOTE, 1987; FELDT; PETERSEN, 2011; AHMED; KHAN; } \\
\text { GHOSH, 2012; MAHANTI; EVANS, 2012; LIM e ANTONY, } \\
\text { 2013; MUJTABA; SHARMA; KHARUB, 2014. }\end{array}$ \\
\hline & $\begin{array}{l}3.4 \quad \text { Interpretação } \\
\text { equivocada das cartas de } \\
\text { controle }\end{array}$ & $\begin{array}{l}\text { MONTGOMERY, 2004; GRIGG e } \\
\text { OAKLAND, 2008; HOERL, SNEE, 2010; COLIN; } \\
\text { VANHOUCKE, 2014; LIM; ANTONY, 2014; SHARMA; } \\
\text { KHARUB, 2014. }\end{array}$ \\
\hline & $\begin{array}{l}3.5 \text { Observação dos } \\
\text { pressupostos estatísticos }\end{array}$ & $\begin{array}{l}\text { SIDDIQUI, 2015; BHOTE; 1987; AHMED; KHAN; GHOSH, } \\
\text { 2012; MUJTABA; FELDT; PETERSEN, 2011; MAHANTI; } \\
\text { EVANS, 2012; LIM e ANTONY, 2013; SHARMA; KHARUB, } \\
\text { 2014; TOLEDO; et.al., 2017. }\end{array}$ \\
\hline & $\begin{array}{l}\text { 3.6 Excesso de ajuste no } \\
\text { processo }\end{array}$ & $\begin{array}{l}\text { SIDDIQUI, 2015; BHOTE; 1987; MUJTABA; FELDT; } \\
\text { PETERSEN, 2011; AHMED; KHAN; GHOSH, 2012; LIM e } \\
\text { ANTONY, 2013; MAHANTI; EVANS, 2012; SHARMA; } \\
\text { KHARUB; 2014. }\end{array}$ \\
\hline & $\begin{array}{l}3.7 \text { Tomada de decisões, } \\
\text { baseada em fatos }\end{array}$ & $\begin{array}{l}\text { BHOTE; 1987; MUJTABA; FELDT; PETERSEN, 2011; } \\
\text { AHMED; KHAN; GHOSH, 2012; MAHANTI; EVANS, 2012; } \\
\text { LIM e ANTONY, 2013; SHARMA; KHARU, 2014; SHPER; } \\
\text { ADLER, } 2017 .\end{array}$ \\
\hline & $\begin{array}{l}3.8 \text { Utilização de } \\
\text { critérios para selecionar } \\
\text { tamanho e frequência de } \\
\text { amostra }\end{array}$ & $\begin{array}{l}\text { BHOTE; 1987; AHMED; KHAN; GHOSH, 2012; MUJTABA; } \\
\text { FELDT; PETERSEN, 2011; MAHANTI; EVANS, 2012; LIM e } \\
\text { ANTONY, 2013, SHARMA; KHARUB, 2014. HARIDY, 2017; } \\
\text { SHPER; ADLER, } 2017\end{array}$ \\
\hline & $\begin{array}{l}\text { 3.9 Implantação de CEP } \\
\text { em processos fora de } \\
\text { controle }\end{array}$ & $\begin{array}{l}\text { BHOTE; 1987; MUJTABA; FELDT; PETERSEN, 2011; } \\
\text { AHMED; KHAN; GHOSH, 2012; MAHANTI; EVANS, 2012; } \\
\text { LIM e ANTONY, 2013; SHARMA; KHARUB; 2014, } \\
\text { SIDDIQUI, } 2015\end{array}$ \\
\hline & $\begin{array}{l}3.10 \text { Falta de revisão } \\
\text { periódica dos limites de } \\
\text { controle }\end{array}$ & $\begin{array}{l}\text { BHOTE; 1987; MUJTABA; FELDT; PETERSEN, 2011; } \\
\text { MAHANTI; EVANS, 2012 AHMED; KHAN; GHOSH, 2012; } \\
\text { LIM e ANTONY, 2013; SHARMA; KHARUB, 2014; } \\
\text { HARIDY, 2017 TOLEDO; et.al., } 2017\end{array}$ \\
\hline$\tilde{J}$ & $\begin{array}{l}\text { 3.11 Estimativa correta } \\
\text { dos limites de controle }\end{array}$ & $\begin{array}{l}\text { BHOTE; 1987; LIM; ANTONY, 2014; SHARMA; KHARUB, } \\
\text { 2014 HARIDY, 2017; TOLEDO; et.al., } 2017 .\end{array}$ \\
\hline 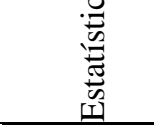 & $\begin{array}{l}3.12 \text { Cálculo e análise } \\
\text { da capacidade do } \\
\text { processo }\end{array}$ & $\begin{array}{l}\text { BHOTE; 1987; MUJTABA; FELDT; PETERSEN, 2011; } \\
\text { AHMED; KHAN; GHOSH, 2012; LIM e ANTONY, 2013; } \\
\text { SHARMA; KHARUB; 2014; TOLEDO; et.al., 2017. }\end{array}$ \\
\hline
\end{tabular}

Fonte: Próprios autores (2018). 
Os fatores que devem ser considerados para uma efetiva implantação do controle estatístico de processo (CEP): uma revisão de literatura

O nível operacional necessita executar as atividades no curto prazo. Em relação à dimensão metodológica, os profissionais devem conhecer os gráficos de controle e outras ferramentas estatísticas básicas para a condução dos projetos de melhoria que está relacionado ao CEP, tais como: Diagrama de Pareto, Histograma e Diagrama de Causa-e-Efeito. Estas ferramentas em especial auxiliam na compreensão de correlações entre as variáveis que estão influenciando a estabilidade do processo produtivo e permitindo maior agilidade na identificação e resolução dos problemas (GRIGG; WALLS, 2007; HOERL; SNEE, 2010).

A utilização de pacotes computacionais é recomendada pela literatura, resultados apontam que a utilização de pacotes computacionais não é utilizada frequentemente pelos operadores em situações do dia a dia. Contudo, dado à necessidade de se desenvolver projetos de melhoria específica, a utilização das ferramentas computacionais torna-se indispensável em razão da complexidade das situações para se trabalhar (SORIANO, 2015).

Ainda em relação à dimensão metodológica, é importante que haja a interpretação adequada das catas de controle para que as ações corretas sejam conduzidas e para essa interpretação é importante que haja conhecimento dos conceitos estatísticos, relacionados ao CEP e, mais especificamente, às cartas de controle (BHOTE, 1987; FELDT; PETERSEN, 2011; AHMED; KHAN; GHOSH, 2012; MAHANTI; EVANS, 2012; LIM ; ANTONY, 2013; MUJTABA; SHARMA; KHARUB, 2014).

Em relação à dimensão estatística, como recomendação básica, a literatura estudada adverte para maior atenção na aplicação do CEP quando da existência de processo fora de controle. Pois, nesta situação as causas especiais de variação podem distorcer os resultados, o que pode levar a resultados equivocados e a uma sucessão de problemas. Majoritariamente, as cartas de controle auxiliam na identificação das causas comuns de variação. Causas assinaláveis apareceram nas cartas de controle, mas por sua natureza a aplicação das cartas de controle não possibilita a identificação das mesmas (CASTAGLIOLA, 2009; 2013; CASTAGLIOLA; MARAVELAKIS， 2009; SHAININ， 1996; WOODALL， 2000; MONTGOMERY; WOODALL, 1999).

A observação dos pressupostos estatísticos, tais como distribuições de probabilidades e noções básicas de estatística como média, moda, mediana, subgrupos racionais e desvio padrão, é importante para assegurar análises e decisões corretas. Neste sentido, compreendem-se noções de distribuição dos dados, saber classificar os parâmetros de processo em atributo ou variável, além de possibilitar a seleção correta da carta de controle, GEPROS. Gestão da Produção, Operações e Sistemas, v. 15, nº 1, p. 71- 91, 2020. 
Os fatores que devem ser considerados para uma efetiva implantação do controle estatístico de processo (CEP): uma revisão de literatura

seu correto preenchimento e interpretação (CASTAGLIOLA, 2009; 2013; CASTAGLIOLA; MARAVELAKIS， 2009; SHAININ， 1996; WOODALL， 2000; MONTGOMERY; WOODALL, 1999; BHOTE; 1987; HAYES; ROMIG, 1977; MONTGOMERY, 2010; ASQ/AIAG, 2005).

Os operadores devem observar tendências gráficas de deslocamento do processo para que sejam tomadas providências no processo, visando à retomada do seu nível de estabilidade, antes da necessidade de paradas e do surgimento de problemas mais graves, permitindo a decisão baseada em fatos (CASTAGLIOLA, 2009; 2013; CASTAGLIOLA; MARAVELAKIS, 2009; SHAININ， 1996; WOODALL， 2000; MONTGOMERY; WOODALL, 1999). Os operadores devem de estar aptos para lidar com seleção de tamanho e frequência amostral a ser utilizado, assim como, devem tomar ações que promovam a revisão periódica dos limites de controle, estimar corretamente os limites de controle e a capacidade do processo, do contrário, as decisões baseadas nas cartas de controle serão equivocadas (MAHANTI; EVANS, 2012 AHMED; KHAN; GHOSH, 2012; LIM; ANTONY, 2013; SHARMA; KHARUB, 2014; HARIDY, 2017; TOLEDO et.al., 2017).

A análise correta da capacidade do processo produtivo possibilita evidenciar a necessidade do uso das cartas de controle e a frequência de coleta de dados além de fornecer elementos para estimar a probabilidade da produção de itens defeituosos pelo processo. Um dos principais problemas derivados da violação de pressupostos estatísticos consiste no ajuste desnecessário do processo produtivo. Muitos ajustes podem ocorrer de maneira equivocada, levando a consequências desastrosas e aumento da variabilidade. É importante que o operador se baseie em fatos, ao tomar decisões de alterar algum parâmetro do processo (ASQ/AIAG, 2005 CASTAGLIOLA, 2009; 2013; CASTAGLIOLA; MARAVELAKIS, 2009; SHAININ, 1996; WOODALL, 2000; MONTGOMERY; WOODALL, 1999; BHOTE; 1987; HAYES ; ROMIG, 1977; MONTGOMERY, 2010; SORIANO; OPRIME; LIZARELLI, 2017).

\section{CONSIDERAÇÕES FINAIS}

O CEP, como conjunto de ferramentas para a melhoria da qualidade, presta uma valorosa contribuição para as subsequentes implantações de abordagens de melhoria tais 
Os fatores que devem ser considerados para uma efetiva implantação do controle estatístico de processo (CEP): uma revisão de literatura

como: o Seis Sigma, Kaizen e o Lean Manufacturing, pois seus conceitos servem de base para materializar as iniciativas e projetos de melhoria (SORIANO; OPRIME; LIZARELLI, 2017).

Os FCS associados ao CEP são descritos e explorado na literatura, contudo, há poucos trabalhos que descrevam, por níveis hierárquicos, as reponsabilidades em relação aos FCS e como estas afetam o processo de implantação. Assim, os responsáveis podem acreditar que são os únicos responsáveis pelo sucesso do programa, quando na verdade a chave para o sucesso do projeto de implantação consiste na delegação de responsabilidades por todos os níveis (SORIANO; OPRIME; LIZARELLI, 2017).

De fato, a efetividade do processo de implantação consiste em reconhecer as múltiplas dimensões envolvidas e delegar corretamente as atividades aos seus corretos níveis de decisão. A literatura estudada demonstra a existência de trabalhos que enfatizam algumas dimensões (DOES; TRIP; SCHIPPERS, 1997; ELG; OLSSON; DAHLGAARD, 2008; FIRKA, 2011).

Alguns trabalhos, tais como Bhote (1987), Burr (1976), Hayes e Romig (1997), valorizam a dimensão estatística e, desta forma, constituem fontes de conhecimento estatístico básico para aqueles que buscam a implantação do CEP em suas organizações. Outros trabalhos, a exemplo de Anghel (2001), ASQ/AIAG (2005), Basilevsky (1994), Grigg; Walls (2007) Juran; Godfrey (1999), Kolarik (1995), Montgomery (2004), Oakland (2008) além de valorizarem a dimensão estatística, tendem a indicar aspectos metodológicos para a condução das atividades de implantação. Tais autores observam os aspectos estatísticos pertinentes, porém consideram que está dimensão não é a única responsável pelo sucesso da redução da variabilidade no processo produtivo.

Observa-se por meio da literatura estudada que em um segundo momento histórico os autores perceberam que apenas a parte estatística do CEP não representa de fato o sucesso do programa. Nesta segunda fase, a literatura apresenta casos de sucesso e como devem ser conduzido às praticas do programa para uma efetiva implantação do programa. Assim os trabalhos de Ahmad (2012), Antony; Balbontin e Taner (2000), Gordon (1994), Mahanti; Evans (2012); Phyanthamilkumaran; Fernando (2008), Putri; Yusof (2009), Rohani; Yusof; Mohamad (2010), Rungasamy; Antony e Ghosh (2002), Rupa; Evans (2012), por exemplo, focam questões gerenciais, apresentando os principais fatores que podem impactar o programa e como tais problemas podem ser minimizados. 
Os fatores que devem ser considerados para uma efetiva implantação do controle estatístico de processo (CEP): uma revisão de literatura

Este trabalho tem implicações práticas e acadêmicas, em relação às implicações acadêmicas um estudo que compila os FCS e os divide em dimensões de aplicações e níveis organizacionais e permite que estudos subsequentes sobre CEP possam utilizar essa visão geral ou mesmo se aprofundar em uma única dimensão e/ou nível hierárquico. Em relação às implicações práticas, há implicações que permitem que a alta administração observe os FCS de cada nível hierárquico para direcionar suas ações de implantação do CEP, assim como o nível tático pode compreender as duas responsabilidades e as do nível operacional, permitindo a criação de treinamentos direcionados para o atendimento dos FCS do nível operacional.

Como trabalhos futuros, verifica-se a carência de estudos que mostrem, efetivamente, como as decisões do nível estratégico e mesmo as do nível tático interferem no resultado da implantação do CEP. Diante do exposto, verifica-se a necessidade de explorar questões relacionadas à infraestrutura ideal para a condução das atividades, dado que a literatura estudada não é clara ao delegar atividades aos níveis tático, estratégico e gerencial da organização. Apesar do grande número de FCS apontados, não se sabe quais são mais críticos, já que não há estudos empíricos sobre o assunto, o que pode trazer benefícios para gerentes e pesquisadores.

Este trabalho reuniu os FCS descritos na literatura para a implantação do CEP que são pertinentes a todos os ramos de atividades econômica, independentemente do tipo de carta de carta de controle que a empresa venha a utilizar ou mesmo o tipo de produto e processo que a organização trabalhe. Contudo, aplicações em setores de atividade econômicas não tradicionais podem revelar novos fatores que precisam ser estudados caso a caso e futuramente incorporado ao estado da arte atual e podem haver ênfases em Fatores específicos dependendo do setor de atividade, além da inclusão de Fatores específicos para a atividade da empresa.

Como limitações deste trabalho destaca-se que apesar do número extenso de fatores que os gestores devem observar para uma efetiva implantação do CEP listados e captados pela revisão de literatura podem ocorrer outros decorrentes das atividades empíricas e da área de atuação da organização. Também há limitações de interpretação, já que existem situações nas quais os fatores não estão citados diretamente pelos autores, assim apenas com uma leitura atenta do trabalho puderam ser observados e alguns fatores citados neste trabalho são definidos de forma diferente, porem descritos de maneira semelhante em trabalhos distintos. 
Os fatores que devem ser considerados para uma efetiva implantação do controle estatístico de processo (CEP): uma revisão de literatura

\section{Referências}

RAHMAN, M. N. A.; ZAIN, R. M.; ALIAS, A. M.; NOPIAH, Z. M. Statistical process control: Best practices in small and medium enterprises. Maejo International Journal of Science and Technology, v. 9, n. 2, p. 193-208, 2015.

AHMADAN, M.F; ZAKUAN. N.; JUSOH, A.; TAKALAC, J. Relationship of TQM and business performance with mediators of SPC, lean production and TPM. Procedia-Social and Behavioral Sciences, v. 65, p. 186-191, 2012.

AHMED, A.; KHAN, I.; GHOSH, M. K. SPC Implementation in Pharmaceutical Industry for Material Flow Management. Interscience Management Review (IMR), ISSN, p. 2231-1513, 2012.

ANGHEL, C. Statistical Process Control Methods from the Viewpoint of Industrial Application. Economic Quality Control, v. 16, n. 1, p. 49-63, 2001.

ANTONY, J.; BALBONTIN, A.; TANER. Key ingredients for the effective implementation of statistical process control. Work study, v. 49, n. 6, p. 242-247, 2000.

ANTONY, J; TANER, T. A conceptual framework for the effective implementation of statistical process control. Business Process Management Journal, v. 9, n. 4, p. 473- 489, 2003.

ASQ/AIAG Measurement Systems Analysis Reference Manual, 3 ed., 2002

ASQ/AIAG Statistical Process Controls (SPC) Reference Manual, ed. 2005

BASILEVSKY, A. Statistical Factor Analysis and Related Methods: Theory and Applications. New York: Wiley Interscience, 1994.

BEVILACQUA, M. GIACCHETTA, G; CIARAPICA, F. E.; MARCHETTI, B. Implementation of a quality procedure based on Delphi method and the ISO/TS 16949: 2009 in the production of stainless steel tubes for automotive exhaust systems. International Journal of Quality \& Reliability Management, v. 28, n. 8, p. 841-866, 2011.

BHOTE, K. R. SPC Made Easier, Simpler, More Statistically Powerful. Target, v. 3, 1987.

BRERETON, P.; KITCHENHAM, B. A.; BUDGEN, D.; TURNER, M.; KHALIL, M. Lessons from applying the systematic literature review process within the software engineering domain. Journal of systems and software, v. 80, n. 4, p. 571-583, 2007.

BURR, I. W. Statistical quality control methods. New York: Marcel Dekker, c1976. 522 p.

CASTAGLIOLA, P.; ACHOURI, A. TALEB, H; CELANO, G.; PSARAKIS, S. Monitoring the coefficient of variation using control charts with run rules. Quality Technology and Quantitative Management, v. 10, n. 1, p. 75-94, 2013.

CASTAGLIOLA, P.; CELANO, G.; CHEN, G. The exact run length distribution and design of the S2 chart when the in-control variance is estimated. International Journal of Reliability, Quality and Safety Engineering, v. 16, n. 01, p. 23-38, 2009.

CASTAGLIOLA, P.; MARAVELAKIS, P. E. A CUSUM control chart for monitoring the variance when parameters are estimated. Journal of Statistical Planning and Inference, v. 141, n. 4, p. 1463$1478,2011$.

COLIN, J.; VANHOUCKE, M. Setting tolerance limits for statistical project control using earned value management. Omega, v. 49, p. 107-122, 2014.

CONFORTO, E. C.; AMARAL, D. C.; SILVA, S. L. DA. Roteiro para revisão bibliográfica sistemática: aplicação no desenvolvimento de produtos $\mathrm{e}$ gerenciamento de projetos. In: CONGRESSO BRASILEIRO DE GESTÃO DE DESENVOLVIMENTO DE PRODUTO, n. 8. Anais... CNGDP 2, n. 1998, p. 1-12, 2011. 
Os fatores que devem ser considerados para uma efetiva implantação do controle estatístico de processo (CEP): uma revisão de literatura

DOES, R. J. M. M.; TRIP, A.; SCHIPPERS, W. A. J. A framework for implementation of statistical process control. International Journal of Quality Science, v. 2, n. 3, p. 181-198. 1997.

ELG, M.; OLSSON, J.; DAHLGAARD, J. Implementing statistical process control: an organizational perspective. International Journal of Quality \& Reliability Management, v. 25, n. 6, p. 545-560, 2008.

FIRKA, D. Statistical, technical and sociological dimensions of design of experiments. The TQM Journal, v. 23, n. 4, p. 435-445, 2011.

FLORAC, W.; CARLETON, A., BARNARD, J. Statistical process control: analyzing space shuttle onboard software process. Software, IEEE, v. 17, n. 4, p. 97-106, 2000.

GORDON, M. E.; PHILPOT, J.W.; BOUNDS, G. M.; LONG, W.S. Factors associated with the success of the implementation of statistical process control. The Journal of High Technology Management Research, v. 5, n. 1, p. 101-121, 1994.

GRAÇA, M.; GOMES, P.; LAGES, L. F.; ZULEMA, L. P. The role of TQM in strategic product innovation: an empirical assessment. International Journal of Operations \& Production Management, v. 34, n. 10, p. 1307-1337, 2014.

GRIGG, N.; WALLS, L. The role of control charts in promoting organizational learning: New perspectives from a food industry study. The TQM Magazine, v. 19, n. 1, p. 37-49, 2007.

GROOVER, M. P. Automation, production systems, and computer-integrated manufacturing. Prentice Hall Press, 2007.

HARIDY, S.; MAGED, A.; KAYTBAY, S.; ARABY, S. Effect of sample size on the performance of Shewhart control charts. The International Journal of Advanced Manufacturing Technology, v. 90, n. 1-4, p. 1177-1185, 2017.

HAYES, G. E.; ROMIG, H. G. Modern quality control. Encino: Bruce, c1977. 849 p.

HOERL, R. W.; SNEE, R. D. Closing the gap. Quality Progress, v. 43, n. 5, p. 52-53, 2010.

HRADESKY, J. L. Aperfeiçoamento da qualidade e da produtividade: guia prático para a implementação do controle estatístico de processos-CEP. McGraw-Hill, 1989.

JALOTE, P.; SAXENA, A. Optimum control limits for employing statistical process control in software process. Software Engineering, IEEE Transactions on, v. 28, n. 12, p. 1126-1134, 2002.

LEVINSON, W. A. Statistical Process Control for Real-World application; CRC Press, 2011.

LIM, S.A.; ANTONY, J.; HE, Z.; ARSHED, N. Critical observations on the statistical process control implementation in the UK food industry. International Journal of Quality \& Reliability Management, v. 34, n. 5, p. 684-700, 2017.

LIM, S. A. H.; ANTONY, J.; ALBLIWI, S. Statistical Process Control (SPC) in the food industry-A systematic review and future research agenda. Trends in Food Science \& Technology, v. 37, n. 2, p. 137-151, 2014.

LIM, S.A.H.; ANTONY, J. A conceptual readiness framework for statistical process control (SPC) deployment. In: INDUSTRIAL ENGINEERING AND ENGINEERING MANAGEMENT, (IEEM). Anais... IEEE International Conference on. IEEE, 2013. p. 300-304.

LIM, S.A.H.; ANTONY, J.; ARSHED, N. A Critical Assessment on SPC Implementation in the UK Food Industry. Journal of Systemics, v. 14, n. 1, p. 37-42, 2016.

LIZARELLI, F. L.; BESSI, N.C.; OPRIME, P.C.; AMARAL R. M.; CHAKRABORTI, S. A bibliometric analysis of 50 years of worldwide research on statistical process control. Gestão \& Produção, v. 23, n. 4, p. 853-870, 2016. 
Os fatores que devem ser considerados para uma efetiva implantação do controle estatístico de processo (CEP): uma revisão de literatura

MAHANTI, R.; EVANS, J. R. Critical success factors for implementing statistical process control in the software industry. Benchmarking: An International Journal, v. 19, n. 3, p. 374-394, 2012.

MONTGOMERY, D. C. A modern framework for achieving enterprise excellence. International Journal of Lean Six, v. 1, n.1, p. 56-65, 2010.

MONTGOMERY, D. C. Design and analysis of experiments. 6 ed. New York: John Wiley, 2005. $643 \mathrm{p}$.

MONTGOMERY, D. C. Introdução ao controle estatístico da qualidade. 4 ed. Rio de Janeiro: LTC, 2014. 513p.

MUJTABA, S.; FELDT, R.; PETERSEN, K. Analyzing strategy and processes for product customization in large-scale industrial settings. In: SOFTWARE ENGINEERING AND ADVANCED APPLICATIONS (SEAA), 2011, 37 ${ }^{\text {th }}$. Anais... EUROMICRO Conference on. IEEE, 2011. p. 369373.

NOSKIEVICOVA, D.; PAUCHOVA, A.; KELBLEROVA, M. Analysis of effective implementation of SPC. Annals of DAAAM \& Proceedings, 2011.

OAKLAND, J. S. Statistical process control. Routledge, 2008.

PHYANTHAMILKUMARAN S.Z; FERNANDO Y. The role of cultural change in the relationship between critical factors with the success of Statistical Process Control (SPC) projects. Proceedings of Annual London Conference on Money, Economy and Management, 3-4 Temmuz 2008, Imperial College, London.

PUTRI, N. T.; YUSOF, S. M. Critical success factors for implementing quality engineering tools and techniques in malaysian's and indonesian's automotive industries: An Exploratory Study. Proceedings of the International Multi Conference of Engineers and Computer Scientists. 2009. p. 18-20.

RANTAMÄKI, J.; TIAINEN, E. L.; KÄSSI, T. A case of implementing SPC in a pulp mill. International Journal of Lean Six Sigma, v. 4, n. 3, p. 321-337, 2013.

ROHANI, J.M.; YUSOF, S.M.; MOHAMAD, I. The Development of a Survey Instrument for Measuring a Relationship Between Statistical Process Control Success Factors and Performance. Journal Mekanikal, n. 30, p. 1-16, 2010.

ROWLEY, J.; SLACK, F. Conducting a literature review. Management research news, v. 27, n. 6, p. 31-39, 2004.

RUNGASAMY, S.; ANTONY, J.; GHOSH, S. Critical success factors for SPC implementation in UK small and medium enterprises: some key findings from a survey. The TQM Magazine, v. 14, n. 4, p. 217-224, 2002.

RUPA M., EVANS, J.R. Critical success factors for implementing statistical process control in the software industry, Benchmarking: An International Journal, v. 19, n. 3, p. 374 - 394, 2012.

SANTANA Jr. M.B. Pesquisa ação sobre os fatores de sucesso para a implantação e continuidade do uso de cartas de controle estatístico de processo em uma planta química no Brasil. 2014. 87f. Dissertação (Mestrado em Pós-Graduação em Engenharia de Produção) UFSCar, São Carlos, 2014.

SHAININ, D. Cartas de controle vs Pré-controle: carroças vs Avião a Jato. In: BHOTE, Keki R. Qualidade de classe mundial. Rio de Janeiro: Qualitymark, 1996. 183-208.

SHARMA, R.; KHARUB, M. Attaining competitive positioning through SPC-an experimental investigation from SME. Measuring Business Excellence, v. 18, n. 4, p. 86-103, 2014.

SHPER, V.; ADLER, Y. The importance of time order with Shewhart control charts. Quality and Reliability Engineering International, v. 33, n. 6 p.1169 - 1177, 2017. 
Os fatores que devem ser considerados para uma efetiva implantação do controle estatístico de processo (CEP): uma revisão de literatura

SIDDIQUI, Y. A.; SAIF, A.; CHEDED, L.; RAHIM, A. Integration of multivariate statistical process control and engineering process control: a novel framework. The International Journal of Advanced Manufacturing Technology, v. 78, n. 1-4, p. 259-268, 2015.

SNEE, R. D. Statistical thinking and its contribution to total quality. American Statistician, p. 116$121,1990$.

SORIANO, F. R.; OPRIME, P. C. Utilização de técnicas multivariadas em projetos Seis Sigma. São Paulo-SP: NEA, 2014. 180p.

SORIANO, F. R.; OPRIME, P. C.; LIZARELLI, F. L. Impact analysis of critical success factors on the benefits from statistical process control implementation. Production, v. 27, 2017.

SORIANO, F. R. Análise da aplicação do controle estatístico de processo segundo as percepções dos usuários do setor de autopeças. 2015. Tese (Doutorado em Engenharia de Produção) UFSCar, São Carlos, 2015.

TOLEDO, J. C.; LIZARELLI, F. L.; SANTANA-JR. M. Success factors in the implementation of statistical process control: action research in a chemical plant. Production, v. 27, 2017.

WOODALL, W. H. Controversies and contradictions in statistical process control. Journal of Quality Technology, v. 32, n. 4, p. 341-350, 2000.

XIE, M.; GOH, T. N. Statistical techniques for quality. The TQM magazine, v. 11, n. 4, p. 238-242, 1999. 